\title{
Estimaciones de la distribución espacial del riesgo relativo de mortalidad por las principales zoonosis en Chile: enfermedad de Chagas, hidatidosis, síndrome cardiopulmonar por hantavirus y leptospirosis
}

\author{
Rodolfo Reyes ${ }^{1,2}$, Karla Yohannessen ${ }^{1}$, Salvador Ayala ${ }^{3}$ y Mauricio Canals,
}

Background: Zoonoses are infections caused by all types of etiological transmissible agents from vertebrate animals to humans. During the last decades, the risk to health caused by different zoonoses has been a consequence of the natural distribution of the different etiological agents and by the emergence and reemergence of these diseases. Aim: To study the distribution of the risk of mortality of the four main zoonoses in continental Chile, based on national mortality data, with the objective of visualizing geographically where to focus the control efforts of these diseases. Methods: Relative risk was estimated by means of Bayesian Statistics. Results: The distribution in Chile of the main zoonoses was obtained. Discussion/Conclusion: The risk maps obtained show a parasitic disease transmitted by high-risk vectors in the north, Chagas disease; a parasitic disease of biological communities in which man is an accidental host, associated with livestock areas, more prevalent in the south, hydatidosis; a bacterial disease transmitted by vertebrates, especially by rodents, where water is an important vehicle, dominant in the center, leptospirosis; and a viral disease transmitted by rodents, very dominant in the south, the hantavirus infection.

Keywords: Zoonoses; distribution; risk; Chagas disease; South American trypanosomiasis hydatidosis; hantavirus infection; leptospirosis.

Palabras clave: Zoonosis; distribución; riesgo; enfermedad de Chagas; tripanosomiasis; hidatidosis; infección por hantavirus; leptospirosis.

\section{Introducción}

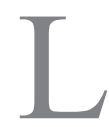
as zoonosis son enfermedades o infecciones causadas por todo tipo de agentes etiológicos (bacterias, parásitos, hongos, virus y agentes no convencionales) transmisibles desde animales vertebrados a humanos y viceversa ${ }^{1}$. Durante las últimas décadas, el riesgo para la salud ocasionado por diferentes zoonosis ha sido generado por la distribución natural de los distintos agentes etiológicos y por la emergencia y re-emergencia de estas enfermedades ${ }^{2,3}$.

A nivel global se estima que existen más de mil microorganismos patógenos conocidos que afectan al hombre, de los cuales hasta $65 \%$ son de origen animal y zoonóticos ${ }^{3}$, los que, en su mayoría necesitan de un reservorio silvestre como condición esencial para su persistencia en una determinada área geográfica ${ }^{1}$. La transmisión de un agente zoonótico, está dado por distintos mecanismos como son el contacto directo (vía ingestión o inhalación), por vectores intermediarios o mordeduras y en ciertos casos por una combinación de éstos. La transmisión de una infección zoonótica entre seres humanos, es inusual, aunque existen algunos agentes que pueden ser transmitidos por transfusión de derivados sanguíneos o trasplante de órganos o tejidos ${ }^{4}$. Las zoonosis reúnen una serie de características que propician su diseminación, complican su control y eventual erradicación. Entre las más importantes se encuentran las siguientes ${ }^{5}$ :

- Algunos de los agentes zoonóticos pueden infectar a una amplia variedad de especies animales.

- En la mayoría de los casos se trata de enfermedades de curso crónico, con tendencia a permanecer latentes o en fase subclínica durante un largo tiempo.

- El 80\% los patógenos zoonóticos tiene la capacidad de afectar a diferentes especies de animales.

- Los animales silvestres representan un riesgo potencial, dado que existe evidencia de que $70 \%$ de las zoonosis tienen su origen en estos animales y se considera que son los responsables de la persistencia e incluso la reemergencia de múltiples zoonosis.
'Programa de Salud Ambiental, Instituto de Salud Poblacional, ESP, Facultad de Medicina, Universidad de Chile. Santiago, Chile.

2Programa Magister de Salud Pública, Instituto de Salud Poblacional, ESP, Facultad de Medicina, Universidad de Chile. Santiago, Chile.

${ }^{3}$ Sub-departamento de Vigilancia de Laboratorio, Instituto de Salud Pública de Chile (ISP). Santiago, Chile.

${ }^{4}$ Departamento de Medicina, Facultad de Medicina (O), Universidad de Chile. Santiago, Chile.

Conflictos de interés: Los autores declaran no tener conflictos de interés.

Financiamiento: Fondecyt

1150514.

Recibido: 29 de mayo de 2019 Aceptado: 3 de septiembre de 2019

Correspondencia a: Mauricio Canals Lambarri mcanals@uchile.cl 
Este tipo de enfermedades se encuentra diseminadas en todo el planeta y $43,6 \%$ de los agentes etiológicos que las producen presentan distribución mundial ${ }^{6}$, donde aproximadamente 13 enfermedades presentan un alto impacto poblacional, provocando más de 2.400 millones de casos en humanos con 2,2 millones de fallecidos. Estas enfermedades corresponden a: leptospirosis, cisticercosis, tuberculosis (forma zoonótica), rabia, leishmaniasis, brucelosis, hidatidosis (equinococosis quística), toxoplasmosis, fiebre Q, tripanosomosis, hepatitis E y ántrax ${ }^{7}$.

Por otra parte, en los últimos años las enfermedades zoonóticas han ido cobrando mayor importancia en salud humana y animal. Esto debido a los nuevos comportamientos y modos de vida adoptados por la población, lo que, sumado a la expansión del comercio internacional, al crecimiento demográfico y al desequilibrio del medio ambiente, entre otros factores, han permitido que distintos agentes zoonóticos puedan adaptarse, transformarse y trasladarse a nuevos hospedadores y ecosistemas ${ }^{8,9}$. Todo lo anterior, ha favorecido la aparición y persistencia de zoonosis, incrementando con esto el riesgo de que el ser humano esté expuesto a estos patógenos. Por esta razón, no sólo seguirán apareciendo nuevas enfermedades zoonóticas, sino que además sus manifestaciones clínicas se presentarán de forma más rápida, afectando a un mayor número de poblaciones y nuevas áreas geográficas ${ }^{8,9}$.

Según la información estadística y epidemiológica existente en el país, es posible apreciar que, en comparación con otros países de la región (Sur y Centroamérica), las enfermedades zoonóticas de importancia en salud pública se encuentran relativamente controladas, lo que se ha logrado a través de la articulación de programas de vigilancia y control de salud humana y animal, por la implementación de campañas de educación sanitaria en poblaciones de riesgo, entre otros. Sin embargo, la realidad es diferente dado que, cada vez más, estas enfermedades aparecen en zonas geográficas nuevas o re-emergen en lugares donde ya habían sido erradicadas, lo que se transforma en un importante problema sanitario, tanto en la salud humana como animal ${ }^{1}$. Muchas enfermedades zoonóticas son sub-notificadas y hay pocas investigaciones recientes del riesgo epidemiológico de estas enfermedades, con excepción de algunos estudios referentes a la enfermedad de Chagas ${ }^{10}$.

En este trabajo estudiamos la distribución del riesgo de mortalidad de las cuatro principales zoonosis en Chile continental, basados en datos nacionales de mortalidad, con el objetivo de visualizar geográficamente donde focalizar los esfuerzos de control de estas enfermedades.

\section{Material y Métodos}

Se desarrolló un estudio ecológico de la mortalidad nacional generada por enfermedades zoonóticas de noti- ficación obligatoria (NO), incluidas en el decreto 158 de 2004 del Ministerio de Salud "Reglamento Sobre Notificación de Enfermedades Transmisibles de Declaración Obligatoria" entre los años 1997 y 2016.

El estudio se realizó sobre todo el territorio nacional, utilizando todas las causas de muerte generadas anualmente y según regiones, de las cuatro zoonosis prevalentes incluidas en el reglamento de notificación de enfermedades transmisibles de declaración obligatoria, las que corresponden a hidatidosis (CIE-10: B67), leptospirosis (CIE-10: A27), enfermedad de Chagas (CIE-10: B57) y hantavirosis (CIE-10: B334, J128 y A985) durante los años 1997 a 2016 en el país. Se consideró este período de tiempo porque, a partir del año 1997, se cuenta con datos oficiales de mortalidad codificados mediante CIE-10 y hasta el año 2016 porque es la última base de datos de mortalidad publicada oficialmente en la página web del Departamento de Estadísticas e Información de Salud (DEIS). Las variables de estudio fueron obtenidas desde las bases de datos de defunciones de los años 1997 a 2016 del (DEIS), siendo las variables incluidas y analizadas diagnóstico, defunciones (número), año y comuna. La comuna fue la unidad de análisis. También se utilizó la base de datos de proyecciones poblacionales del INE, donde se obtuvo la población nacional estimada según datos oficiales por comuna y año. Toda la información utilizada fue de acceso público y descargada desde los sitios web http://www.deis.cl/y https://ine.cl/estadisticas/ censos/censos-de-poblacion-y-vivienda

Los datos se registraron en hojas de cálculo Excel y el análisis estadístico se llevó a cabo utilizando el software estadístico Stata 14. Para cumplir con los objetivos, los mapas Bayesianos de riesgo relativo se construyeron utilizando el modelo Besag-York-Mollie (BYM) ${ }^{1}{ }^{1}$ utilizando el software WinBUGS complementado con ArcGIS. El riesgo relativo mide los casos observados en relación con los esperados por el tamaño de la población y con las variaciones espaciales y temporales aleatorias. Se obtuvo la distribución espacial del riesgo relativo de cada zoonosis. El modelo BYM asume que el número de casos $\left(O_{i t}\right)$ en el área i y el período t sigue una distribución de Poisson con media $\mu_{i t}=e_{i t} * r_{i t}$, con $r_{i t}=$ riesgo relativo y $e_{i t}=$ número esperado de casos. Es decir, $\log \left(\mu_{i}\right)=$ $\log \left(e_{i t}\right)+\alpha+\theta_{i t}$, donde $\alpha$ es un parámetro constante (tasa global), y $\theta_{i t}=S_{i t}+U_{i t}$. $U_{i t}$ representa la fluctuación aleatoria no espacial, con una distribución Normal $(0$, $\left.\sigma_{v}^{2}\right)$, y $S_{i t}$ es la variabilidad espacialmente estructurada, es decir la influencia de las zonas vecinas (estructura de vecindades) en el riesgo relativo de una localidad (comuna). Estos efectos espaciales aleatorios se modelan con una distribución condicional auto-regresiva (CAR) cuya distribución conjunta es $\sim \mathrm{N}\left(0, \sigma_{U}^{2} Q-\right)$, donde $\mathrm{Q}$ es la matriz que define la estructura de vecindad ${ }^{11}$. Finalmente, el riesgo relativo viene dado por: $r_{i t}=e_{i t} \alpha+\theta_{i t}$. El número 


\begin{tabular}{|c|c|c|c|c|c|c|c|c|}
\hline \multirow[b]{2}{*}{ Región } & \multicolumn{2}{|c|}{ Enfermedad de Chagas } & \multicolumn{2}{|c|}{ Hidatidosis } & \multicolumn{2}{|c|}{ Leptospirosis } & \multicolumn{2}{|c|}{ Hantavirosis } \\
\hline & Tasa & $\mathbf{R R}$ & TASA & RR & TASA & RR & TASA & RR \\
\hline Arica y Parinacota & $0.32 \pm 0.64$ & $1.63 \pm 0.68$ & $0.00 \pm 0.00$ & $0.67 \pm 0.26$ & $0.00 \pm 0.00$ & $0.62 \pm 0.19$ & $0.00 \pm 0.00$ & $0.59 \pm 0.24$ \\
\hline Tarapacá & $0.50 \pm 0.89$ & $1.43 \pm 0.81$ & $0.02 \pm 0.06$ & $0.92 \pm 0.35$ & $0.00 \pm 0.00$ & $0.62 \pm 0.17$ & $0.00 \pm 0.00$ & $0.59 \pm 0.20$ \\
\hline Antofagasta & $0.40 \pm 0.86$ & $1.61 \pm 1.32$ & $0.01 \pm 0.03$ & $0.82 \pm 0.19$ & $0.00 \pm 0.00$ & $0.57 \pm 0.15$ & $0.00 \pm 0.00$ & $0.57 \pm 0.20$ \\
\hline Atacama & $4.39 \pm 6.76$ & $5.38 \pm 5.98$ & $0.00 \pm 0.00$ & $0.78 \pm 0.17$ & $0.00 \pm 0.00$ & $0.69 \pm 0.12$ & $0.00 \pm 0.00$ & $0.58 \pm 0.15$ \\
\hline Coquimbo & $11.14 \pm 8.38$ & $15.43 \pm 11.89$ & $0.15 \pm 0.35$ & $1.49 \pm 1.37$ & $0.00 \pm 0.01$ & $0.77 \pm 0.25$ & $0.00 \pm 0.00$ & $0.56 \pm 0.18$ \\
\hline Valparaíso & $1.50 \pm 3.53$ & $2.68 \pm 5.32$ & $0.08 \pm 0.33$ & $0.85 \pm 0.82$ & $0.02 \pm 0.07$ & $1.23 \pm 1.16$ & $0.01 \pm 0.06$ & $0.60 \pm 0.31$ \\
\hline Metropolitana & $0.11 \pm 0.19$ & $0.31 \pm 0.29$ & $0.02 \pm 0.07$ & $0.50 \pm 0.45$ & $0.01 \pm 0.03$ & $0.46 \pm 0.45$ & $0.02 \pm 0.06$ & $0.38 \pm 0.32$ \\
\hline O'Higgins & $0.25 \pm 1.16$ & $0.65 \pm 1.21$ & $0.00 \pm 0.00$ & $0.71 \pm 0.18$ & $0.00 \pm 0.00$ & $0.76 \pm 0.17$ & $0.24 \pm 0.89$ & $1.26 \pm 1.74$ \\
\hline Maule & $0.00 \pm 0.00$ & $0.26 \pm 0.10$ & $0.29 \pm 0.91$ & $1.56 \pm 1.84$ & $0.08 \pm 0.21$ & $2.93 \pm 3.60$ & $0.22 \pm 0.55$ & $1.60 \pm 1.81$ \\
\hline Ñuble & $0.39 \pm 1.80$ & $0.44 \pm 0.70$ & $0.40 \pm 1.80$ & $1.42 \pm 1.51$ & $0.13 \pm 0.27$ & $3.45 \pm 4.01$ & $0.55 \pm 1.44$ & $2.17 \pm 2.39$ \\
\hline Bío-Bío & $0.00 \pm 0.00$ & $0.21 \pm 0.10$ & $0.56 \pm 1.79$ & $1.69 \pm 2.41$ & $0.04 \pm 0.08$ & $1.82 \pm 1.55$ & $0.39 \pm 1.46$ & $1.71 \pm 1.89$ \\
\hline La Araucanía & $0.00 \pm 0.00$ & $0.25 \pm 0.07$ & $0.58 \pm 1.14$ & $2.71 \pm 2.93$ & $0.00 \pm 0.00$ & $0.77 \pm 0.18$ & $0.65 \pm 1.50$ & $2.83 \pm 3.41$ \\
\hline Los Ríos & $0.00 \pm 0.00$ & $0.23 \pm 0.05$ & $1.09 \pm 1.74$ & $4.47 \pm 4.56$ & $0.00 \pm 0.01$ & $0.94 \pm 0.32$ & $0.72 \pm 1.67$ & $2.47 \pm 2.54$ \\
\hline Los Lagos & $0.00 \pm 0.00$ & $0.32 \pm 0.17$ & $0.43 \pm 1.86$ & $1.57 \pm 2.15$ & $0.00 \pm 0.00$ & $0.78 \pm 0.15$ & $0.64 \pm 2.88$ & $1.64 \pm 2.08$ \\
\hline Aysén & $0.00 \pm 0.00$ & $0.57 \pm 0.19$ & $0.17 \pm 0.52$ & $2.16 \pm 2.60$ & $0.00 \pm 0.00$ & $0.86 \pm 0.10$ & $3.64 \pm 8.91$ & $4.58 \pm 5.59$ \\
\hline Magallanes & $0.00 \pm 0.00$ & $0.58 \pm 0.20$ & $0.18 \pm 0.49$ & $1.73 \pm 1.08$ & $0.00 \pm 0.00$ & $0.85 \pm 0.14$ & $0.00 \pm 0.00$ & $0.90 \pm 0.19$ \\
\hline
\end{tabular}

esperado de casos se estimó como $e_{i t}=P_{i t} I_{t}$, donde $P_{i t}$ es la población total de la comuna e $I_{t}$ es el promedio de muertes reportadas para cada zoonosis por cada $100 \mathrm{mil}$ habitantes en Chile en el período considerado.

\section{Resultados}

El modelo BYM tuvo una adecuada convergencia para las estimaciones usando 5.000 iteraciones, permitiendo una adecuada estimación de los riesgos relativos. Las tasas de mortalidad (riesgos absolutos) y los riesgos relativos estimados por región se muestran en la Tabla 1 y los mapas de riesgos por comuna en las Figuras 1 a la 4.

\section{Discusión}

Los fallecidos constituyen una muestra restringida del total de casos de una enfermedad, ya que no todos los enfermos mueren. La mortalidad es un mejor estimador de la carga de una enfermedad en la población en el caso de enfermedades de alta letalidad. En el caso particular de las zoonosis estudiadas, para la enfermedad de Chagas no existen estimaciones de la letalidad. En pacientes no tratados, en la fase aguda de la enfermedad, no reportada en Chile, tiene letalidades entre 2 y $12 \%{ }^{12}$, pero en el Chagas crónico es muy difícil estimarla. Como referencia, de los infectados, aproximadamente 20 a $25 \%$ desarrollarán una cardiopatía o megadigestivos y de éstos, 5 a $10 \%$ tendrán una cardiopatía grave, potencialmente mortal $^{13}$. Para la hidatidosis se reporta una letalidad de
$1,3 \%{ }^{14}$; para el síndrome cardiopulmonar por hantavirus entre 30 y $47 \%{ }^{15}$ y para leptospirosis entre 5 y $20 \%{ }^{16}$. Es decir, incluimos en este estudio enfermedades que van desde baja a alta letalidad. Sin embargo, las tasas de mortalidad tienen la virtud de ser basadas en certificados de defunción que consignan el diagnóstico, apoyados en estudios clínicos y de laboratorio, constituyendo un dato fidedigno, aunque restringido de la carga de una enfermedad en una localidad ${ }^{17,18}$ que permite evaluar la efectividad de las intervenciones de salud pública, en términos de acceso, calidad y oportunidad ${ }^{19}$.

Las estimaciones de riesgo relativo, es decir la razón entre los casos observados y los esperados por el tamaño poblacional son mejores estimadores que las tasas de mortalidad por cien mil habitantes ya que estas últimas, aunque miden el riesgo absoluto observado en una localidad, es fuertemente influido por el tamaño poblacional de la localidad ${ }^{10}$. Por ejemplo, un solo fallecido en una localidad de mil personas corresponde a la altísima tasa de 100/100 mil habitantes; lo que muestra que la tasa de mortalidad sobre-estima el riesgo en comunas pequeñas. El modelo BYM usado en este trabajo para la estimación del riesgo relativo tiene la virtud de incorporar el efecto de las localidades vecinas en la estimación del riesgo de una enfermedad, lo que se conoce como el efecto de la estructura de vecindades. Es decir, una localidad puede tener riesgo, aunque no haya casos, si las localidades cercanas los tienen ${ }^{11}$.

La hidatidosis es una zoonosis parasitaria producida por larvas de los céstodos del género Echinococcus, del 


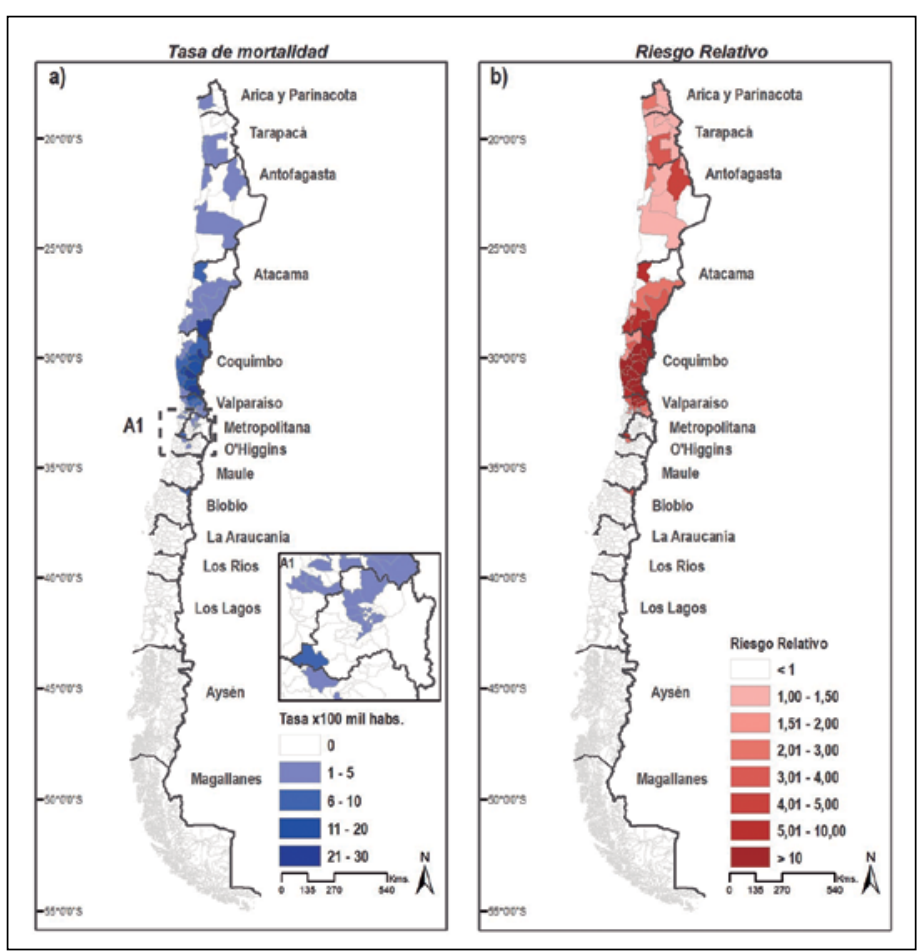

Figura 1. Distribución de las tasas (a) y del riesgo relativo de mortalidad (b) de la enfermedad de Chagas en Chile.

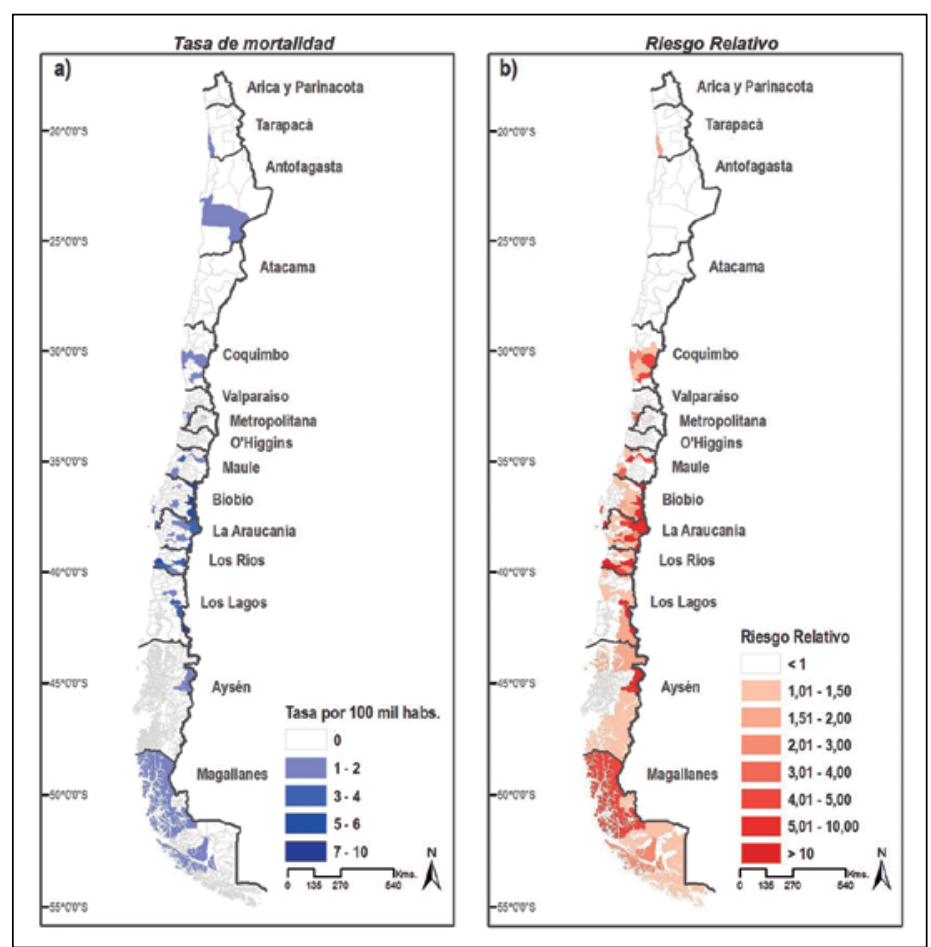

Figura 2. Distribución de las tasas (a) y del riesgo relativo de mortalidad (b) de la hidatidosis en Chile.

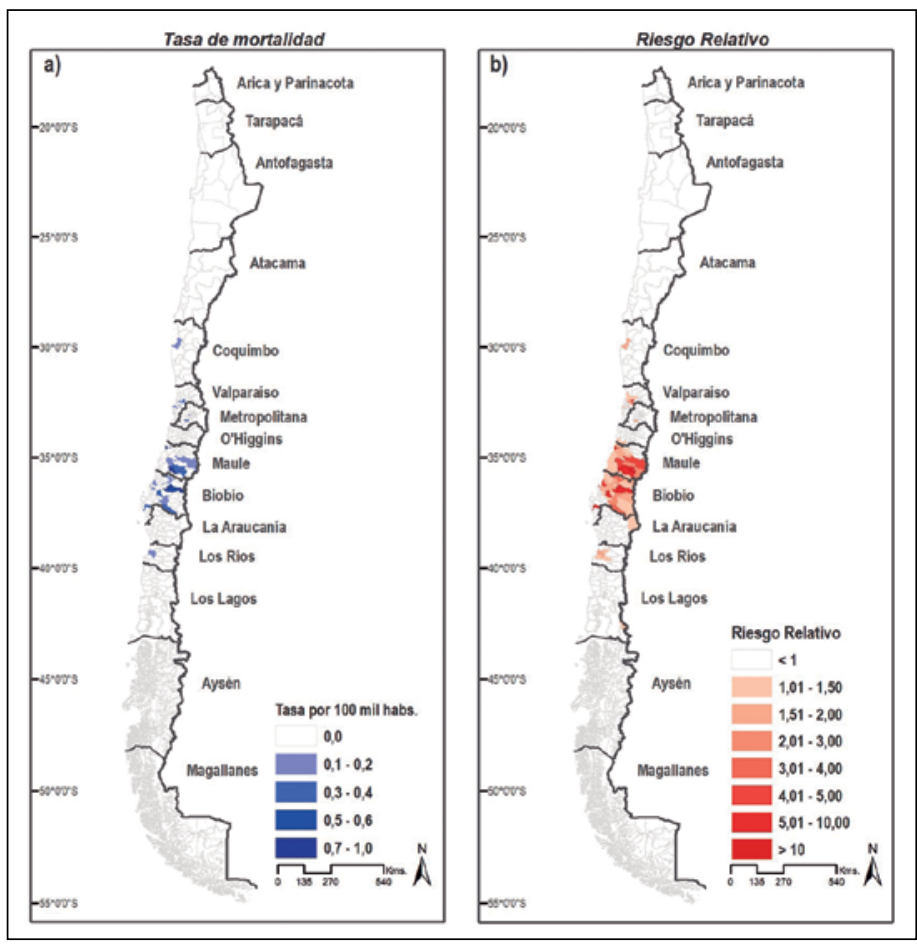

Figura 3. Distribución de las tasas (a) y del riesgo relativo de mortalidad (b) de la leptospirosis en Chile.

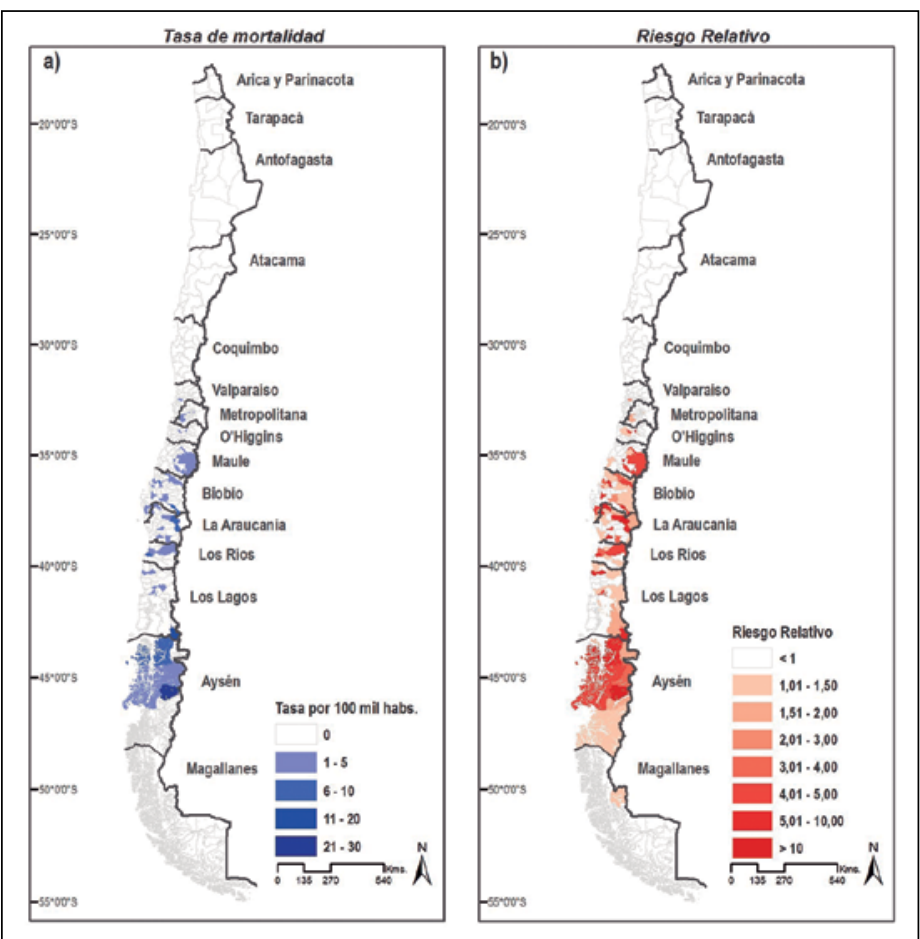

Figura 4. Distribución de las tasas (a) y del riesgo relativo de mortalidad (b) del síndrome cardiopulmonar por hantavirus en Chile. 
que se existen cuatro especies relevantes: E. granulosus, E. multilocularis, E. vogeli y E. oligarthrus, siendo E. granulosus el principal responsable de la enfermedad (equinococosis quística) en humanos y la única especie demostrada en Chile. Todas las especies presentan una distribución mundial con mayores prevalencias en zonas templadas que incluyen países mediterráneos, Rusia, Asia central y China y algunas regiones de Australia y América (especialmente Sudamérica) ${ }^{20}$. En el mundo se estima que la enfermedad afecta a entre 2 y 3 millones de personas, existiendo aproximadamente 18.000 casos nuevos por año ${ }^{20,21}$. Presenta una tasa de mortalidad de 2 a $4 \%$, la que puede aumentar al no existir un tratamiento adecuado. Por otra parte, si la enfermedad se presenta en el pulmón y no existe tratamiento o es limitado, la mortalidad puede superar el $90 \%$ después de 10-15 años del diagnóstico de la enfermedad ${ }^{21}$. En Chile, la enfermedad tiene un carácter endémico, en especial en zonas rurales dedicadas al pastoreo ovino. Según el sistema de notificación ENO, los casos de hidatidosis humana han variado de 297 en el año 2001 (52,2\% mujeres) a 267 en 2009 (52,4\% hombres), con un promedio anual de 311 casos $^{22}$. Las tasas de notificación se han desplazado desde 1,9 casos por 100 mil hab., en 2001 a 1,6 casos en 2009, evidenciando una tendencia al decrecimiento. Respecto de la distribución geográfica, la mayor tasa de casos reportados se registra en Aysén con 28,9 casos por 100 mil hab., en el año $2009^{22}$. Por otra parte, la mortalidad por hidatidosis presenta una disminución en el tiempo, siendo la segunda causa de muerte por enfermedad parasitaria, después de la enfermedad de Chagas, con 30 a 40 defunciones anuales y una tasa de mortalidad que varía entre 0,09 y 0,21 por cada 100 mil hab. entre los años 2000 y 2012 a nivel nacional, concentrándose en áreas urbanas de las regiones del Maule, Araucanía, los Lagos y Aysén ${ }^{22-24}$. Los estimadores nacionales consideran una tendencia al decrecimiento; sin embargo, no dejan ver las realidades regionales, donde no sólo existe una distribución geográfica heterogénea asociada a la economía básica, evidenciada por el aumento de los casos a medida que se avanza hacia el sur, si no también, por las diferencias en la calidad y acceso a las prestaciones de salud ${ }^{22}$.

Martínez P. ${ }^{25}$, en un estudio entre 2000-2010, encontró las mayores tasas de mortalidad en las regiones de $\mathrm{La}$ Araucanía, Aysén, Los Lagos y Maule. Estas regiones tienen también alta representación en nuestro estudio con un intervalo temporal mayor. Los riesgos relativos son más claros en destacar una zona de riesgo desde Maule hacia el sur, que corresponde a la zona ganadera y un foco en la Región de Coquimbo. En esta última región, la prevalencia de equinococosis canina se ha estimado en $7,2 \%$ y varía entre $5,9 \%$ en caprinos y $23,9 \%$ en bovinos ${ }^{26}$. Así, en esta región, la hidatidosis es favorecida por la existencia de un gran número de animales de abasto en zonas rurales, la pobreza y el menor acceso a los servicios de salud ${ }^{22}$. Recientemente se ha destacado el bajo nivel educacional como relevante, que aumenta el riesgo de adquirir esta enfermedad ${ }^{27}$.

La leptospirosis es una enfermedad infecciosa reemergente, ocasionada por bacterias patógenas del género Leptospira, del cual se conocen más de 250 serovares. Afecta a humanos y animales (domésticos y silvestres) y presentan una distribución mundial, dada la amplia gama de hospederos que posee, que va desde mamíferos salvajes y domésticos hasta reptiles y anfibios, siendo los más importantes los roedores ${ }^{28,29}$. La presentación de la infección es estacional, y la mayoría de los casos aparece entre verano y otoño en regiones templadas, siendo considerada principalmente como una enfermedad ocupacional ${ }^{28,29}$. La infección puede ser adquirida en forma indirecta, mediante el contacto con agua o suelos contaminados, o directa, asociada a la exposición con animales infectados, principalmente roedores, y sus productos (fundamentalmente orina), siendo tanto animales silvestres como domésticos (roedores, ganado, caninos, etc.) hospederos naturales de la infección ${ }^{30}$. Estimaciones de la OMS y de la Sociedad Internacional de Leptospirosis, señalan que los casos anuales en el mundo son alrededor de 350.000 a 500.000, presentándose en Estados Unidos de América (E.U.A.) entre 100 a 200 casos por año. Junto con esto, se estima que la letalidad para la forma no ictérica varía entre 1 y $5 \%$, y en la forma ictérica alcanza hasta $54 \%$ en casos graves con compromiso cardiaco, presentando una mortalidad general de 5 a 15\% ${ }^{28,31}$. En América del Sur, la prevalencia de la enfermedad es desconocida, pero distintos estudios han mostrado prevalencias por serología en humanos (número de positivos/población estudiada) según área geográfica, como, por ejemplo, $80,6 \%$ en Venezuela, 67,9\% en zonas urbanas de Colombia y $48 \%$ y $77,2 \%$ en áreas urbanas y rurales de Brasil, respectivamente $^{30}$. En nuestro país se desconoce la seroprevalencia de la enfermedad en humanos, detectándose entre los años 2003 y 2010 un total de 142 casos, números que han ido disminuyendo de 27 casos en 2003 a 5 en 2010, concentrándose principalmente en las regiones del Maule (3,3 casos por 100 mil hab.), Biobío (2,3 casos por $100 \mathrm{mil}$ hab.), Los Lagos (1,8 casos por 100 mil hab.), Valparaíso (1,4 casos por 100 mil hab.) y Región Metropolitana $(0,3 \text { casos por } 100 \text { mil hab. })^{30}$. La tasa de mortalidad se ha desplazado desde 0,019 muertes por 100 mil hab. en 2003 a 0,024 en 2007, con un total de 17 fallecidos $^{30}$. Nuestro estudio es consistente en señalar, tanto en las tasas como en el riesgo relativo un foco de riesgo que incluye a Maule, Ñuble y Bío-Bío, y un segundo foco en la Región de Valparaíso. En estas zonas, probablemente se conjugan los factores condicionantes de riegos altos de leptospirosis: la presencia de agua, ya sea en forma de lluvias, cursos naturales e inundaciones, que aumentan la 
población y dispersión de la leptospira; la temperatura que favorece la proliferación y la supervivencia; la infraestructura sanitaria como los alcantarillados que pueden inundarse y permitir la dispersión; el contacto del humano con el reservorio animal ${ }^{32}$. Este último caso puede ocurrir en las faenas agrícolas, lo que hace de esta enfermedad un problema laboral. En este sentido, se ha reportado en Chile que los casos se concentran en hombres obreros, asociado a su condición laboral y se ha propuesto que los casos en la Región del Maule podrían tener relación con la producción de arroz, que conjuga la presencia de agua y roedores ${ }^{30}$.

La enfermedad de Chagas, es causada por el protozoo parásito Trypanosoma cruzi. La enfermedad, se encuentra ampliamente distribuida desde el sur de E.U.A. hasta el norte de Chile y Argentina, afectando a 21 países de Latinoamérica, donde $13 \%$ de la población es considerada en riesgo de infección ${ }^{33}$. La enfermedad puede ser transmitida al hombre, a más de 150 especies de animales domésticos y mamíferos silvestres. Para el ser humano, la forma más común de contraer la infección en zonas endémicas es a través del contacto con las deyecciones infectadas del insecto vector, siendo menos probable otras formas de transmisión como la transplacentaria, transfusión sanguínea y trasplantes de órganos, entre otras ${ }^{34}$. Por otra parte, Triatoma infestans corresponde al principal vector domiciliario que transmite la enfermedad en los países del Cono Sur. Actualmente, y según la OMS, la enfermedad afecta, aproximadamente, a entre 8 y 12 millones de personas, con incidencias anuales que varían entre 28.000 y 41.000 afectados en América. Además, se estima que entre 20 y $30 \%$ de los individuos infectados desarrollarán la enfermedad grave, generándose aproximadamente 12.000 muertes al año ${ }^{35,36}$. En México, la incidencia ha variado entre 0,07 casos por cada 100 mil hab. en el año 2000 (mortalidad de $0,02 \%$ ) y 0,7 casos por cada 100 mil hab. en el año 2012 (mortalidad de $0,03 \%$ ) $^{36}$. En nuestro país, el parasito vector (T. infestans), se extiende desde la Región de Arica-Parinacota hasta O'Higgins, certificándose a Chile en el año 1999 como un país que interrumpió la transmisión vectorial por $T$. infestans de la enfermedad ${ }^{36}$. Por otra parte, y durante el período 1990 y 2008, la tasa promedio de notificación de casos se mantuvo estable en 2,95 por 100 mil hab., aumentando en 2009 a 6,79 por 100 mil hab., y a 11,55 en el año $2011^{37}$. La mortalidad por esta enfermedad ha mostrado una leve tendencia al alza, con tasas de 0,3 por 100 mil hab. desde el año 2005 en adelante, y 0,4 por 100 mil hab. en 2011 (tasa promedio: $0,36 \pm 0,55$ ). Durante el período 2006-2011, la mortalidad representó $0,06 \%$ del total de muertes anuales del país ${ }^{36}$. En nuestro estudio la zona de riesgo es clara en esta enfermedad: desde Arica y Parinacota hasta la Región de Valparaíso, la que es coincidente con lo que señalan las tasas de casos notificados, mostrando una buena relación entre notificaciones de casos y defunciones. La zona de riesgo de la enfermedad de Chagas coincide con la zona de distribución del vector doméstico (T. infestans) (18-34 $\left.{ }^{\circ} \mathrm{LS}\right)$ y los vectores silvestres Mepraia gajardoi (18-24 $\left.{ }^{\circ} \mathrm{LS}\right)$ y M. spinolai (25-34 $\left.{ }^{\circ} \mathrm{LS}\right)$. Aunque en los últimos años ha existido una mayor dispersión de los casos explicado por migraciones, esto aún no se manifiesta en las zonas de riesgo de mortalidad.

Los hantavirus (género Hantavirus, familia Bunyaviridae) son un grupo de virus con diferentes antígenos, transportados por roedores y animales insectívoros, de los cuales se han identificado a lo menos 20 variantes, las que pueden causar, una enfermedad leve, moderada o grave. En el caso de Chile, el hospedero y reservorio primario es el roedor silvestre Oligoryzomys longicaudatus (ratón colilarga), en quien se produce una infección crónica con viremia persistente y asintomática, eliminando el virus por la orina, saliva y excretas ${ }^{15}$. El hombre es un hospedero accidental y contrae la infección por contacto con roedores y sus excretas, sobre todo por transmisión aerógena, aunque las mordeduras también pueden generar infección ${ }^{15}$. Estos virus, presentan una distribución mundial y son conocidos como el hantavirus del Viejo Mundo y del Nuevo Mundo, debido a la distribución geográfica de sus reservorios de roedores y al tipo de enfermedad que se manifiesta en humanos ${ }^{38}$. En el ser humano se manifiestan principalmente en dos grandes síndromes: fiebre hemorrágica con síndrome renal (FHSR) y el síndome cardiopulmonar por hantavirus $(\mathrm{SCPH})$, que es provocado por variantes presentes en el continente americano, donde se han documentado casos de SCPH en E.U.A., Canadá, Panamá, Brasil, Paraguay, Bolivia, Chile, Uruguay y Argentina ${ }^{39}$.

El síndrome de FHSR, presenta una seroprevalencia en humanos (presencia de anticuerpos por serología) variable según área geográfica, con zonas como Finlandia con prevalencia de $5 \%$ y regiones donde puede alcanzar a $21 \%$. Por otra parte, Suecia ha informado tasas tan altas como 40\% y en Francia, Alemania, Países Bajos y Bélgica las seroprevalencias en humanos de la variante PUU oscilan entre 1 y $2 \%$. La incidencia anual varía según región y año, donde China declara entre 150.000 y 210.000 casos anuales; Rusia y Corea notifican entre cientos y miles de casos anuales y en el norte de Europa se producen entre 100 y 1.000 casos por año ${ }^{39}$.

En el continente americano, las prevalencias de SCPH varían según la región, con seroprevalencias de infección entre 0,5 y $2 \%$ en E.U.A., Canadá y algunas regiones de Argentina, hasta $40 \%$ descrito en poblaciones indígenas del Chaco Paraguayo. Se ha observado que la enfermedad afecta mayormente a individuos de sexo masculino ${ }^{39}$. La letalidad varía según síndrome y variante del virus; para el caso del FHSR, los virus Dobrava (7-12\%) y virus Hantaan (10-15\%), presentan los mayores porcentajes; 
mientras que, para el SCPH, las mayores mortalidades son producidas por las variantes virus Sin Nombre (40-60\%), el virus Andes (43-56\%) y los virus Muleshoe, Black Creek Canal y Bayou con mortalidad es superiores a $40 \%{ }^{39}$.

En Chile, desde mediados de la década del 90 se han notificado casos del SCPH por el virus Andes, aunque estudios serológicos retrospectivos han demostrado la presencia de anticuerpos contra hantavirus en muestras de pacientes desde el año $1975^{15}$. La enfermedad se ha presentado de forma endémica con un marcado aumento estacional (noviembre a abril) ${ }^{40}$, describiéndose entre los años 1995 y 2012 un total 764 casos de SCPH, distribuidos entre las regiones de Coquimbo y La Araucanía, siendo la Región del Bío-Bío la que concentra el mayor número de casos acumulados (194 casos), seguida por la Región de Los Lagos (134 casos) ${ }^{15}$. Por otra parte, desde el año 1995 y hasta 2010 , se ha reportado un total de 238 fallecidos por la enfermedad, presentando una letalidad que oscila entre 40 y $50 \%$ y una tasa de mortalidad que el año 2010 fue de 0,13 por cada 100 mil hab., duplicando lo registrado durante $2009(0,05 \text { por } 100 \text { mil hab. })^{40-43}$.

Nuestro estudio muestra una clara y continua zona de riesgo entre las regiones de O'Higgins y Aysén. Aunque existen varios roedores que han mostrado seropositividad al virus Andes, como Abrothrix longipilis, A. olivaceus, Phyllotis darwini y Loxodontomys microtus ${ }^{44}$, el reservorio fundamental es $O$. longicaudatus con una tasa de seropositividad entre 2 y $5,9 \%{ }^{45,46}$ y con distribución desde Copiapó (28 ${ }^{\circ} \mathrm{LS}$ ) hasta la Región de Magallanes (55 $\left.{ }^{\circ} \mathrm{LS}\right)$. La explicación para una distribución del riesgo más restringida que la distribución del principal reservorio podría estar asociada a dos factores. Por una parte, las poblaciones de $O$. longicaudatus son más numerosas en esta zona, donde pueden presentarse ratadas asociadas a la cascada trófica lluvias-florecimiento de gramíneas del género Chusquea (quila, colihue), siendo los valores más altos de seropositividad del ratón colilargo medidos durante la ratada de 2011 en la Región de Los Ríos ${ }^{44}$. Por otra parte, la infección por hantavirus en el hombre está, en más de 50\%, asociada a las faenas agrícolas y la ruralidad $^{15}$, actividades que disminuyen drásticamente en la Región Metropolitana con respecto a las zonas más sureñas, explicando el cambio en el riesgo.
Así, los mapas de riesgo obtenidos muestran un panorama interesante, con una enfermedad parasitaria transmitida por vectores de alto riesgo en el norte: la enfermedad de Chagas; una enfermedad parasitaria de comunidades biológicas en que el hombre es un hospedero accidental, asociada a zonas ganaderas, prevalente en el sur: la hidatidosis; una enfermedad bacteriana transmitida por vertebrados, especialmente por roedores, donde el agua es un vehículo importante, dominante en el centro: la leptospirosis; y una enfermedad viral transmitida por roedores, muy dominante en el sur: la hantavirosis.

\section{Resumen}

Introducción: Las zoonosis son enfermedades o infecciones causadas por todo tipo de agentes etiológicos transmisibles desde animales vertebrados a humanos. Durante las últimas décadas, el riesgo para la salud ocasionado por diferentes zoonosis, ha sido generado por la distribución natural de los distintos agentes etiológicos y por la emergencia y reemergencia de estas enfermedades. Objetivo: Estudiar la distribución del riesgo de mortalidad de las cuatro principales zoonosis en Chile continental, basados en datos nacionales de mortalidad, con el objetivo de visualizar geográficamente donde focalizar los esfuerzos de control de estas enfermedades. Metodología: Se estima el riesgo relativo de las principales zoonosis en Chile, mediante estadística Bayesiana. Resultados: Se obtuvo la distribución de las cuatro principales zoonosis de Chile. Discusión/Conclusión: Se obtuvo la distribución de las cuatro principales zoonosis de Chile. Los mapas de riesgo obtenidos muestran una enfermedad parasitaria transmitida por vectores de alto riesgo en el norte, la enfermedad de Chagas; una enfermedad parasitaria de comunidades biológicas en que el hombre es un hospedero accidental, asociada a zonas ganaderas, prevalente en el sur, la hidatidosis; una enfermedad bacteriana transmitida por vertebrados, especialmente por roedores, donde el agua es un vehículo importante, dominante en el centro, la leptospirosis; y una enfermedad viral transmitida por roedores, muy dominante en el sur, la infección por hantavirus.

\section{Referencias bibliográficas}

1.- Cabello C, Cabello F. Zoonosis con reservorios silvestres: Amenazas a la salud pública y a la economía. Rev Med Chile. 2008; 136 : 385-93. http://dx.doi.org/10.4067/S003498872008000300016.

2.- Morse S S. Factors and determinants of disease emergence. Rev Sci Tech. 2004; 23(2): 443-51. PMID: 15702712.

3.- Cobos Valdes D, de Valle Fernández Y,
Labañino N, Martínez W, Peña L, Santos M. Elementos generales para analizar sobre las zoonosis TT. Correo Científico Médico 2014; 18(4): 709-24. http://scielo.sld.cu/pdf/ccm/ v18n4/ccm11414.pdf.

4.- Dabanch J. Zoonosis. Rev Chilena Infectol 2003; 20 (1): 47-51. http://dx.doi.org/10.4067/ S0716-10182003020100008.

5.- Flores Castro R. La situación actual de las zoonosis más frecuentes en el mundo. Gac Med Mex 2010; 146 (6): 423-9. https://www.anmm.
org.mx/GMM/2010/n6/64 vol 146 n6.pdf.

6.- Fuentes M, Pérez L, Suárez Y, Soca M, Martínez A La zoonosis como ciencia y su impacto social. Rev Electrónica Vet. 2006; VII:1-19. http://www.veterinaria.org/revistas/ redvet/n090906.html.

7.- International Livestock Research Institute. Mapping of poverty and likely zoonoses hotspots. Zoonoses Rep. 2012; 1-119. https:// cgspace.cgiar.org/bitstream/handle/10568/21161/ ZooMap_July2012_final.pdf. 
8.- Brown C. Emerging zoonoses and pathogens of public health significance - an overview. Rev Sci Tech Off Int Epiz. 2004; 23 (2): 435-42. PMID: 15702711.

9.- King L J. Zoonosis y patógenos emergentes de importancia para la salud pública. Rev Sci Tech Off Int Epiz. 2004; 23 (2): 429-33. https://web. oie.int/boutique/extrait/contents417422.pdf.

10.- Villa A R, Moreno L, García de la Torre G S Epidemiología y estadística en salud pública. Mc Graw-Hill, España. 2012.

11.- Besag J, York J, Mollie A. Bayesian image restoration with two applications in spatial statistics. Ann Inst Statist Math 1991; 43 (1): 1-59. doi: https://doi.org/10.1007/BF00116466.

12.- Pinto Dias J C, Schofield C J. Social and medical aspects: Morbidity and mortality in general populations. In: Telleria J, Tibayrenc M. American Trypanosomiasis Chagas Disease. One hundred years of research. First Ed. London: Elsevier; 2011. 45-54.

13.- Salvatella R A. Achievements in controlling Chagas disease in Latin America. In Janin J. Revisiting Chagas disease: from a Latin American perspective to a global perspective. WHO; 2007.

14.- Acosta-Jamett G, Vargas R, Ernst S. Caracterización epidemiológica de hidatidosis humana y animal en la Región de Los Ríos, 1999-2009.Rev Chilena Infectol 2016; 33 (4): 419-427. https://scielo.conicyt.cl/pdf/rci/v33n4/ art06.pdf.

15.- Sotomayor V, Aguilera X. Epidemiología de la infección humana por hantavirus en Chile. Rev Chilena Infectol 2000; 17(3): 220-32. http://dx.doi.org/10.4067/S071610182000000300006

16.- Martínez P, Ortega D, Salinas K. Evolución de la leptospirosis según el Sistema de Vigilancia Epidemiológica Nacional, Chile 2003-2009. Rev Chilena Infectol 2012; 29(6): 648-54. http://dx.doi.org/10.4067/S071610182012000700010 .

17.- Gordis L. Epidemiología. Tercera Ed. 2005. Elsevier. Madrid.2005; 336 p.

18.- Bonita R, Beaglehole R, Kjellström T. Epidemiología Básica. Segunda edición. OPS Publicación Científica y Técnica 2008; 629.

19.- Martínez P, Cáceres D, Canals M. Hidatidosis: un problema no resuelto en Chile. Parasitol Latinoam 2016; 65 (3): 20-9. http:// sociedadchilenaparasitologia.cl/wp-content/ uploads/2018/04/PLA-65-3.pdf.

20.- Armiñanzas C, Gutiérrez-Cuadra M, Fariñas MC. Hidatidosis: Aspectos epidemiológicos, clínicos, diagnósticos y terapéuticos. Rev Esp Quimioter. 2015; 28(3): 116-24. https://seq.es/ seq/0214-3429/28/3/farinas.pdf.

21.- McManus D P, Gray D J, Zhang W, Yang Y. Diagnosis, treatment, and management of echinococcosis. BMJ. 2012; 344: e3866-e3866. Disponible en: http://www.bmj.com/cgi/ doi/10.1136/bmj.e3866.

22.- Martínez P. Caracterización de la mortalidad por hidatidosis humana: Chile, 20002010. Rev Chilena Infectol 2014; 31(1): 7-15. http://dx.doi.org/10.4067/S071610182014000100001.

23.- Ministerio de Salud de Chile. Manual para el diagnóstico, tratamiento, prevención y control de la hidatidosis en Chile. 2015. Disponible en: https://diprece.minsal.cl/wrdprss_minsal/wpcontent/uploads/2016/02/ManualHidatidosis.pdf.

24.- Organización Panamerica de la Salud (OPS). Zoonosis Salud Animal. Organización Panamerica de la Salud (OPS). 2017.

Disponible en: https://www.paho.org/chi/index. php?option $=$ com content $\&$ view $=$ article $\&$ id $=19$ 2:zoonosis-salud-animal\&Itemid $=1005$.

25.- Martínez P. Factores climáticos, ambientales antropogénicos y socioeconómicos/ demográficos, en la incidencia de hidatidosis en Chile (2001-2011): antecedentes para la formulación de políticas públicas de gestión ambiental en zoonosis. Tesis Doctor Salún Pública. Facultad de Medicina. Universidad de Chile. 2017.

26.- Soto-Aguilar A, Junod T, Campillay M, Acosta-Jamett G, Landaeta-Aqueveque C. Análisis de la hidatidosis humana en la Región de Coquimbo entre los años 2008 y 2012. Rev Med Chile. 2017; 145: 603-9. http://dx.doi. org/10.4067/S0034-98872017000500007.

27.- Medina N, Rodríguez J, Aguirre O, Riquelme N, Ayala S, Canals M. Distribución y factores de riesgo de hidatidosis en la Región de O’Higgins. Rev Chilena Infectol 2019; (in press). 2019.

28.- García R, Reyes A, Hernández D, Ramírez M, Rivas B. Leptospirosis; un problema de salud pública. Rev Latinoam Patol Clínica 2013; 60 (1): 57-70. https://www.medigraphic.com/pdfs/ patol/pt-2013/pt131g.pdf.

29.- Higgins R. Emerging or re-emerging bacterial zoonotic diseases: bartonellosis, leptospirosis, Lyme borreliosis, plague. Rev Sci Tech 2004; 23 (2): 569-81. PMID: 15702720.

30.- Martínez P, Ortega D, Salinas K. Evolución de la leptospirosis según el Sistema de Vigilancia Epidemiológica Nacional, Chile 2003-2009. Rev Chilena Infectol 2012; 29 (6): 648-54. http://dx.doi.org/10.4067/S071610182012000700010.

31.- The Center for Food Security and Public Health C. Leptospirosis. Clin Microbiol Rev. 2005; 1-8. http://www.cfsph.iastate.edu/Factsheets/es/ leptospirosis-es.pdf.

32.- Lau C L, Smythe L D, Craig S B, Weinstein P. Climate change, flooding, urbanization and leptospirosis: fueling the fire? Trans R Soc Trop Med Hyg 2010; 104 (10): 631-8. doi: 10.1016/j. trstmh.2010.07.002.

33.- Cucunubá Z M, Okuwoga O, Basáñez M-G, Nouvellet P. Increased mortality attributed to Chagas disease: a systematic review and metaanalysis. Parasit Vectors. 2016; 9 (1): 42. doi: 10.1186/s13071-016-1315-x

34.- Murcia L, Carrilero B, Saura D, Iborra M
A, Segovia M. Diagnóstico y tratamiento de la enfermedad de Chagas. Enferm Infecc Microbiol Clin 2013; 31(1): 26-34. doi: 10.1016/S0213-005X(13)70111-3

35.- Molina I, Salvador F, Sánchez-Montalvá A. Actualización en enfermedad de Chagas. Enferm Infecc Microbiol Clin. 2016; 34(2): 132-8. doi: 10.1016/j.eimc.2015.12.008.

36.- Ministerio de Salud de Chile. Norma general técnica control y prevención nacional de la enfermedad de Chagas. Santiago; 2014. 1-98. https://www.minsal.cl/sites/default/files/ NORMA\%20TECNICA_CHAGAS_FINAL. pdf.

37.- Canals M, González C, Canals L, Canals A, Cáceres D, Alvarado S, et al. ¿Qué dicen los números de la evolución temporal de la enfermedad de Chagas? Rev Chilena Infectol 2017; 34 (2): 120-7. http://dx.doi.org/10.4067/ S0716-10182017000200004.

38.- Jonsson CB, Figueiredo LTM, Vapalahti O. A global perspective on hantavirus ecology, epidemiology, and disease. Clin Microbiol Rev. 2010; 23 (2): 412-41. doi: 10.1128/ CMR.00062-09.

39.- Enria DAM, Levis SC. Zoonosis virales emergentes: las infecciones por hantavirus Introducción e historia. Rev Sci Tech Off int Epiz 2004; 23 (2): 595-611. PMID: 15702722.

40.- Navarrete M, Hott M, Caroca J, Leyton L, Venegas N, Ismail K. Correlación entre criterios clínicos y de laboratorio de casos notificados por sospecha de hantavirosis y el resultado de la técnica de referencia. Rev Chilena Infectol 2016; 33 (3): 275-81. https://scielo.conicyt.cl/ $\mathrm{pdf} / \mathrm{rci} / \mathrm{v} 33 \mathrm{n} 3 /$ art04.pdf.

41.- Sotomayor V. Aguilera X. hantavirus en Chile. Rev Chilena Infectol 2000; 17: 220-32. http://dx.doi.org/10.4067/S071610182000000300006.

42.- Ministerio de Salud. Boletín Epidemiológico de Hantavirus [Internet]. Santiago; 2011. Disponible en: http://www.cruzroja.cl/files.

43.- Sotomayor V, Fuenzalida F. Vigilancia epidemiológica de la enfermedad por hantavirus en los años 2009 y 2010. El Vigía 2012; 13 (27): 50-4. http://www.enfermeriaaps. com/portal/wp-content/uploads/2015/06/ vigia27.pdf.

44.- Ferrés M, Palma R E. Epidemiología del Hantavirus en Chile. En: Canals M, Cattan P E. Zoología médica III. Vertebrados. Santiago: Editorial Universitaria; 2012, pp: 123-31.

45.- Jaksíc F M, Lima M. Myths and facts on "ratadas": bamboo blooms, rainfalls peacks and mouse outbreaks. Austral Ecol. 2003; 28: 237-51. https://doi.org/10.1046/j.14429993.2003.01271.x

46.- Medina R A, Torres-Pérez F, Galeno H, Navarrete M, Vial P A, Palma R E, et al. Ecology, genetic diversity, and phylogeographic structure of Andes virus in humans and rodents in Chile. J Virol 2009; 83(6): 2446-59. doi: 10.1128/JVI.01057-08. 